\title{
Supercrystallization of $\mathrm{NaCl}$ from Solution Irradiated by Soft X-Rays
}

\author{
A.J. JanaviČIUS ${ }^{a, *}$, R. PURlys ${ }^{b}$ And R. RinkŪNAS ${ }^{b}$ \\ ${ }^{a}$ Šiauliai University, Faculty of Nature Sciences, P. Višinskio St. 19, 76351 Šiauliai, Lithuania \\ ${ }^{b}$ Vilnius University, Faculty of Physics, Sauletekio Av. 9, LT-10222 Vilnius, Lithuania
}

(Received July 1, 2011; in final form April 20, 2012)

\begin{abstract}
It was found that irradiation of a water solution of $\mathrm{NaCl}$ with the diffractometer DRON3-M (Russian device) had a large influence on two-step processes of crystallization. The irradiation in the first stage of crystallization of the solution produces metastable radicals of water and excited seeds, which stimulate a very fast crystallization after switching off irradiation. After the crystals reach a sufficient size, the crystal growth can be explained by creation of vacancy-interstitial pairs in the growing crystal due to irradiation. The increase of linear dimensions of the growing irradiated crystals is proportional to the square root $\sqrt{t}$ of crystallization time because most important place in crystallization takes the ions diffusion by irradiated vacancies.
\end{abstract}

DOI: $10.12693 /$ APhysPolA.123.777

PACS: 81.10.Dn, 64.70.kp, 32.80.Hd

\section{Introduction}

Crystallization from solution can be considered to be a two-step process [1]. The first step is a phase separation and is called nucleation. The second step is the growth of nuclei to crystals. The requirement for crystallization is supersaturation of solutions, which are not at equilibrium. Every system tends to equilibrium and the transition of a supersaturated solution to the equilibrium state proceeds by stochastic processes of nucleation and crystallization. The relation between the nucleation and crystal growth defines the crystal size distribution. The purity of crystals is strongly dependent on the growth rate. Fast growth may lead to liquid inclusions. For these reasons, the control of crystallization rate is necessary. The industrial crystallization processes require constant rate of crystallization, which can be achieved by measuring supersaturation and by temperature control. This method does not make it possible to achieve an exactly constant rate of crystal growth, because supersaturation level depends on crystal growth, cooling profiles and cooling rate [1]. Stochastic processes depend not only on temperature and diffusion processes but also on generation of charged radicals and superdiffusion of impurities by irradiation [2-5]. In this paper, we will consider influence of irradiated vacancies in $\mathrm{NaCl}$ crystals on its crystallization from water solution. Multiple ionization of $\mathrm{Cl}^{-}$and $\mathrm{Na}^{+}$ions by X-rays is the reason of vacancy formation [6] by a Varley-type mechanism based on the Auger effect. At a time of about $10^{-13} \mathrm{~s}$, which is longer than the period of lattice oscillations, the generated perturbed electrostatic potential can transport $\mathrm{Cl}^{-}$ion into an interstitial state, whence it can diffuse through the

*corresponding author; e-mail: AYanavy@gmail.com crystal. The anion vacancy trapping an electron forms an $\mathrm{F}$ center [6, 7] (an $\mathrm{F}$ center is a vacancy in the halide sublattice with a trapped electron) taking part in crystal coloration [6] and crystallization. Irradiation can also create $\mathrm{H}$ centers [7] (an $\mathrm{H}$ center is an interstitial halide ion with a trapped hole). It is known that heavy irradiation by fast electrons causes formation of large vacancy voids, chlorine bubbles and sodium colloids. F centers facilitate diffusion of $\mathrm{Na}^{+}$and $\mathrm{Ca}^{+}$ions by vacancies, and increase crystallization rate.

Crystallization rate can also be increased in a water solution previously saturated with free radicals [8] by X-ray irradiation.

\section{Production of free radicals in $\mathrm{NaCl}$ water solution by X-rays}

Action of ionizing radiation upon water produces free radicals and electrons [8]. X-rays can change ion charge in solution by the Auger effect [8] and cause various reactions. We consider only the ones that are most important for crystallization. The photoelectric effect can initiate dissociation reactions of water molecules

$$
\mathrm{H}_{2} \mathrm{O} \rightarrow \mathrm{H}+\mathrm{OH}, \quad \mathrm{H}_{2} \mathrm{O} \rightarrow \mathrm{H}^{+}+\mathrm{OH}^{-},
$$

production of hydrated electrons $\mathrm{e}_{\mathrm{a}}$ and unstable radicals $\mathrm{H}_{2} \mathrm{O}^{+}$:

$$
\mathrm{H}_{2} \mathrm{O}+h \nu \rightarrow \mathrm{H}_{2} \mathrm{O}^{+}+\mathrm{e}_{\mathrm{a}}+h \nu_{f}, \quad \nu_{f}<\nu .
$$

$\mathrm{H}_{2} \mathrm{O}^{+}$decomposes within $10^{-13} \mathrm{~s}$ and produces weakly bound complex $\mathrm{H}_{3} \mathrm{O}^{+}$or metastable radicals [8]:

$$
\mathrm{H}_{2} \mathrm{O}^{+}+\mathrm{H}_{2} \mathrm{O} \rightarrow \mathrm{H}_{3} \mathrm{O}^{+}+\mathrm{OH} \text {. }
$$

The metastable radicals $\mathrm{H}_{3} \mathrm{O}^{+}$consist of a neutral molecule $\mathrm{H}_{2} \mathrm{O}$ and a proton $\mathrm{H}^{+}$interacting by covalent bonds, electrostatic forces and the Van der Waals bonds [8], which have a longer range proportional to $1 / r^{7}$. Those metastable radicals $\cdot \mathrm{H}_{3} \mathrm{O}^{+}$collect $\mathrm{Na}^{+}$and $\mathrm{Cl}^{-}$ to metastable nucleus by the Van der Waals and electrical forces. These processes correspond to the following chemical reaction [8]: 


$$
\mathrm{H}_{3} \mathrm{O}^{+}+\mathrm{OH}^{-} \rightarrow 2 \mathrm{H}_{2} \mathrm{O},
$$

photoeffects

$$
\begin{aligned}
& \mathrm{Cl}^{-} \rightarrow \mathrm{Cl}+\mathrm{e}_{\mathrm{a}}, \\
& \mathrm{Na}^{+} \rightarrow \mathrm{Na}^{2+}+\mathrm{e}_{\mathrm{a}}
\end{aligned}
$$

and the Auger effects [9]:

$$
\begin{aligned}
& \mathrm{Cl}^{-} \rightarrow \mathrm{Cl}^{+}+2 \mathrm{e}_{\mathrm{a}}, \\
& \mathrm{Na}^{+} \rightarrow \mathrm{Na}^{+3}+2 \mathrm{e}_{\mathrm{a}} .
\end{aligned}
$$

X-rays produce not only $\mathrm{Na}^{+}, \mathrm{Cl}^{-}$but also ions $\mathrm{Na}^{2+}$, $\mathrm{Na}^{3+}$ and $\mathrm{Cl}^{+}$, charged radicals $\mathrm{H}_{2} \mathrm{O}_{2}^{2-}, \mathrm{H}_{3} \mathrm{O}^{+}, \mathrm{F}$ centers, vacancies in $\mathrm{Na}^{+}$and $\mathrm{Cl}^{-}$sublattices, which affect ions diffusion and surface tension of the crystals taking part in radiation-enhanced crystallization. A very important role can be played by negative $\mathrm{F}$ centers, which enhance diffusion of positive ions $\mathrm{Na}^{+}, \mathrm{Na}^{2+}, \mathrm{Na}^{3+}(6)$, (8) and $\mathrm{Cl}^{+}(7)$ in the growing crystal. The metastable radicals can produce metastable nucleus, which increase crystallization rate in an irradiated solution after switching of X-rays. Crystallization is limited by conservation of charge and by the fact that protons and chlorine ions cannot interact directly. In irradiated solutions, there are excited seeds with complicated chemical and structural configurations, which participate in the fast crystallization. The irradiated solution, like the one in the simple case discussed in [10], must eventually reach the state with the lowest accessible energy. Crystals of especially small sizes are unstable [11] and this instability is increased by irradiation with $\mathrm{X}$-rays. After fast reverse reactions (5), producing negative chlorine ions $\mathrm{Cl}^{-}$and reaching critical size [11], when negative volume energy excites the positive surface energy, the crystals begin to grow with a very large rate. Metastable radicals, irradiated vacancies and ions with large charges and strongest attractive forces can significantly increase the rate of crystallization processes [1].

\section{Crystallization experiments with irradiated solutions}

Crystallization of small $(10-1000 \mu \mathrm{m}) \mathrm{NaCl}$ crystals from a supersaturated non-irradiated and previously irradiated (W anode voltage $30 \mathrm{kV}$, current $20 \mathrm{~mA}$ ) solution in a drying drop placed on a glass was investigated.

Sizes of small crystals were measured by a Russian optical microscope Lumam-IZ and an internet camera for photographing the crystals and measuring distances with a highest resolution of $0.01 \mathrm{~mm}$. An original $\mathrm{C}++$ program, which processes measurement data, can present graphically the kinetics of crystal growth. For crystals with size $1 \mathrm{~mm}$ or greater, we used the in-camera lens Helios-44-2 with a simple exact line and the same $\mathrm{C}++$ program.

We applied soft X-rays emitted by a Russian diffractometer DRON-3M with a $\mathrm{W}$ anode $\left(U_{\mathrm{a}}=30 \mathrm{kV}\right.$, $I_{\mathrm{a}}=20 \mathrm{~mA}$, irradiation time $1 \mathrm{~h}$ ) for irradiation of a supersaturated $\mathrm{NaCl}$ solution in water at $22^{\circ} \mathrm{C}$. The incident X-ray photons cause the Auger effect in $\mathrm{Na}^{+}$ and $\mathrm{Cl}^{+}$, as well as dissociation of water (1) and production of metastable radicals of water molecules. In addition to solvent electrons, the charged metastable radicals $\mathrm{H}_{2} \mathrm{O}_{2}^{2-}, \mathrm{H}_{3} \mathrm{O}^{+}$are produced by chemical reactions. They can produce excited nucleation centers for ions taking part in fast crystal growth. Those metastable radicals participate in slow decomposition and in fast crystallization. The radial crystal growth rate is proportional to diffusion flow of the dissolved material towards the crystal surface. The diffusion in an excited medium irradiated by $\mathrm{X}$-rays can be significantly enhanced. This phenomenon has been named superdiffusion [5].

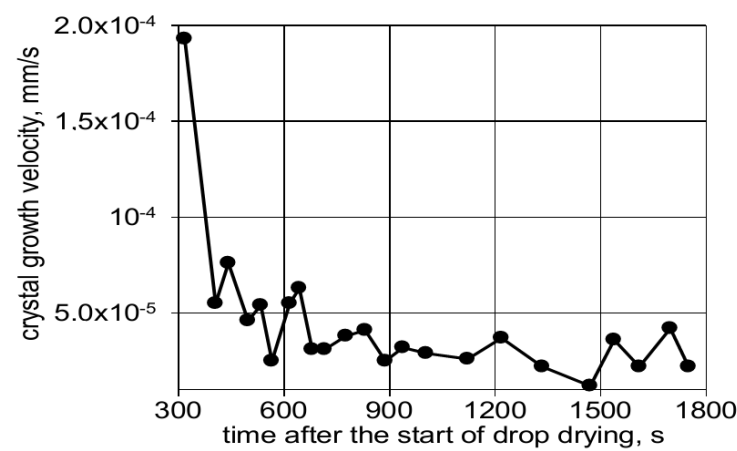

Fig. 1. $\mathrm{NaCl}$ growth rate from water solution dependence on time after the start of drop drying at $22{ }^{\circ} \mathrm{C}$. Growing of crystal in drop was measured from initial size $61 \mu \mathrm{m}$ to $109 \mu \mathrm{m}$.

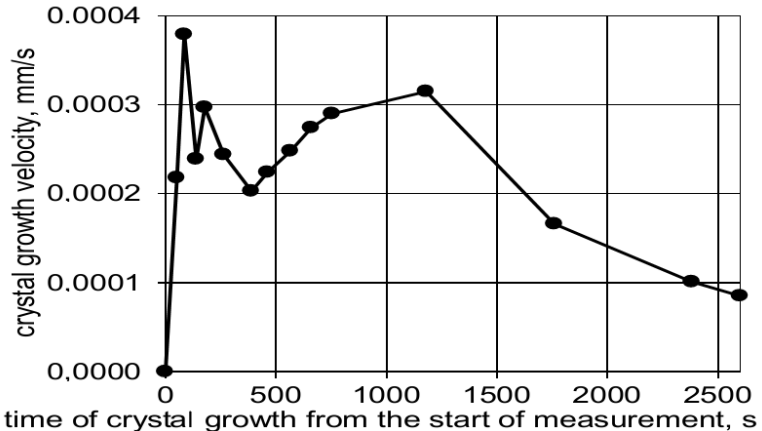

Fig. 2. Time dependence of $\mathrm{NaCl}$ crystal growth rate from water solution at $22^{\circ} \mathrm{C}$ irradiated with X-rays (W anode, voltage $30 \mathrm{kV}$, tube current $20 \mathrm{~mA}$ ). Growing of crystal in drop was measured from $137 \mu \mathrm{m}$ till $279 \mu \mathrm{m}$.

Now we can compare the crystallization processes in the evaporating drops from a simple solution and an irradiated solution. The measured crystallization rates for crystal of initial size $61 \mu \mathrm{m}$ in drop from a supersaturated $\mathrm{NaCl}$ water solution at $22^{\circ} \mathrm{C}$ are presented in Fig. 1 . The rates of crystal growth with the initial sizes about $137 \mu \mathrm{m}$ are presented in Fig. 2 for drop from irradiated solution, where crystallization is at first fast growing and then slows down until $500 \mathrm{~s}$. The crystallization rate 
in irradiated solution in Fig. 2 is at first time very fast growing, because the metastable charged radicals $\mathrm{H}_{2} \mathrm{O}^{+}$, $\mathrm{H}_{3} \mathrm{O}^{+}$and $\mathrm{H}_{2} \mathrm{O}_{2}^{2-}$ initially increase the critical nucleus formation and later crystals growing rate and destination of the close packing of ions in the lattice are coursed by reverse chemical reactions for (2), (3), and (4). From $500 \mathrm{~s}$ to $1500 \mathrm{~s}$, the linear crystallization rate in the drop of irradiated solution of $\mathrm{NaCl}$ presented in Fig. 2 is about 10 times greater than in Fig. 1 for non-irradiated solution. This phenomenon, which is caused by superdiffusion and assisted by metastable charged radicals, vacancies and metastable seeds, will be named "supercrystallization".

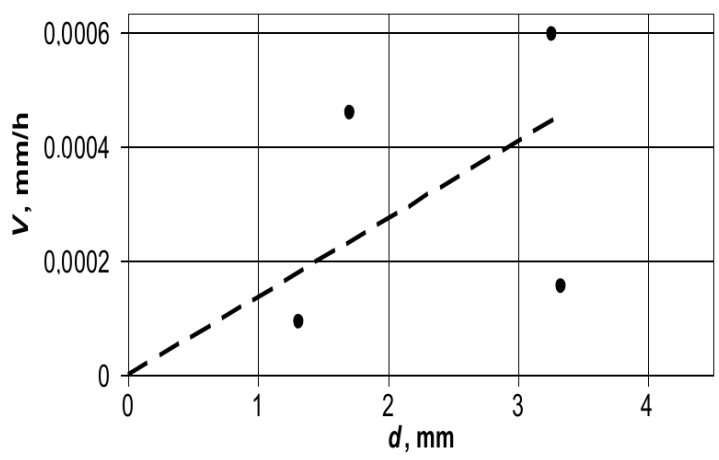

Fig. 3. Dependence of the rate of $\mathrm{NaCl}$ crystal growth from water solution at $22^{\circ} \mathrm{C}$ on initial crystal size.

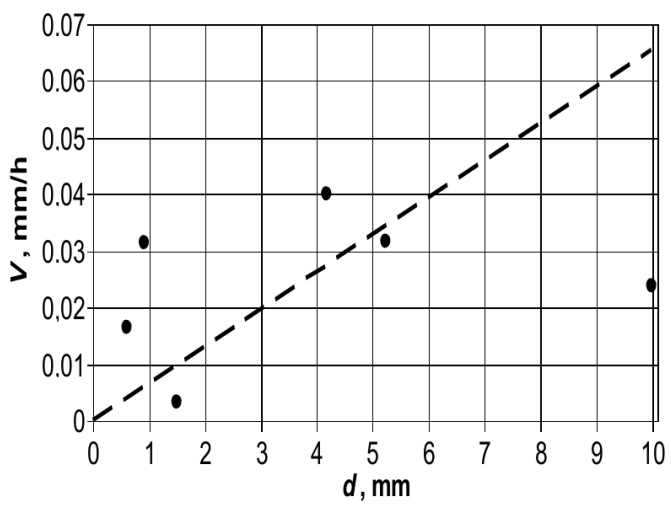

Fig. 4. Dependence of the rate of $\mathrm{NaCl}$ crystal growth from water solution at $22{ }^{\circ} \mathrm{C}$ on initial crystal size under $3 \mathrm{~h}$ time irradiation with X-rays (W anode, $30 \mathrm{kV}$, $20 \mathrm{~mA}$ ).

We provided the measurements of dependence of the crystallization rates on the initial sizes of crystals hanging on the hairs in the cylindrical test-tube with $2 \mathrm{~cm}$ diameter and $4 \mathrm{~cm}$ depth. The results without irradiation and with irradiation are presented in Fig. 3 and Fig. 4. The reason why the linear approximation has been chosen in Fig. 3 is that we cannot achieve a higher accuracy of repeated measurements of growing crystals sizes without changing of crystallization conditions.

The significant increase of crystallization rate was achieved using X-ray irradiation. It was achieved in a certain range of initial size of the crystals. X-ray irradiation prolonged crystallization of those crystals in a supersaturated solution at $20^{\circ} \mathrm{C}$. The comparison of growth rates presented in Fig. 3 and Fig. 4 for crystallization of non-irradiated and irradiated crystals indicates that irradiation causes an increase of linear crystallization rate by a factor 100 and depends on initial sizes on complicated way. The phenomenon of fast growing presented in Fig. 4 is related to superdiffusion of ions from the surface [5] into the irradiated bulk of crystal.

In this case, vacancies, point defects, and induced chemical reactions created by the radiative Auger effect changes Coulomb interactions of electric dipoles or ions with neighboring atoms or ions [10]. The crystal growth rate depends not only on the presence of metastable radicals but also on supersaturation of solution due to an increase of $\mathrm{H}^{+}$concentration [1].

Generation of vacancies in sodium chloride crystals has been achieved by double ionization of sodium cations due to the Auger effect. The resulting positive ions $\mathrm{Na}^{3+}$ displace one of nearest-neighbor chlorine ions by the Coulomb interaction, producing negative, neutral and positive $\mathrm{Cl}$ vacancies [12] in sufficiently large quantities for enhancing ion diffusion in the crystal. Vacancy charge can change spontaneously during energy transitions [11], depending on the charge magnitude. The energy released during transitions between $\mathrm{Cl}$ vacancies [12] of different charge states varies from $3 \mathrm{eV}$ to $5 \mathrm{eV}$ and can generate lattice distortions and relaxations important for diffusion processes. In addition, crystallization depends on concentration of irradiated vacancies, which produce voids in a crystal [7] (concentration $10^{13}-10^{14} \mathrm{~cm}^{-3}$ ) with a radius of about $20-30 \mathrm{~nm}$. They are caused by reactions of divacancy agglomerates filled with chlorine and sodium colloids.

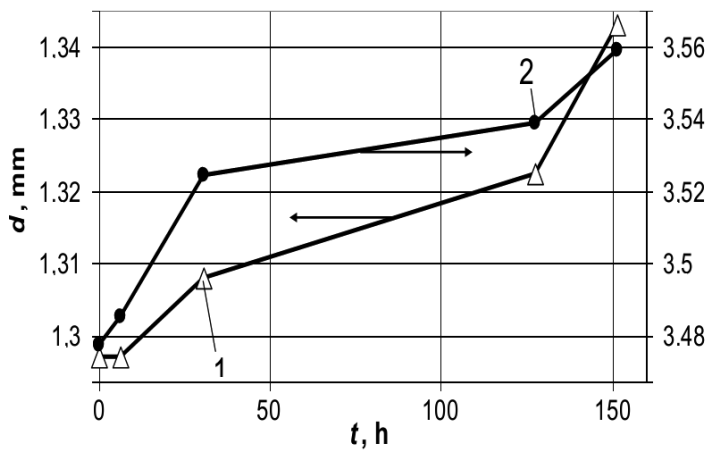

Fig. 5. Time dependence of $\mathrm{NaCl}$ crystal growth starting from different initial sizes $(1.297 \mathrm{~mm}$ (curve 1 ) and $3.477 \mathrm{~mm}$ (curve 2)) in absence of X-ray irradiation.

The dependence of sizes of not irradiated and irradiated crystals was presented in Fig. 5 and Fig. 6 on the initial size and on time $t$ and square root of crystallization time $\sqrt{t}$. It indicates the different character of not irradiated and irradiated crystals growth. Crystallizations rates are characterized by different diffusions flows. 


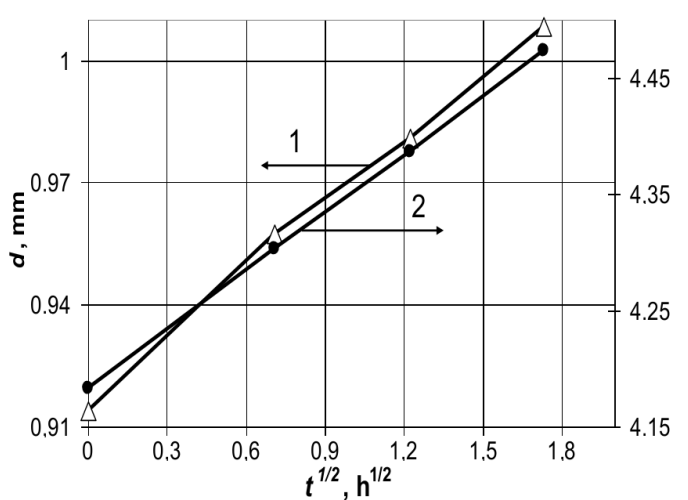

Fig. 6. Dependence of $\mathrm{NaCl}$ crystal growth on $\sqrt{t}$ in the case of two initial sizes $(0.914 \mathrm{~mm}$ (line 1$)$ and $4.183 \mathrm{~mm}$ (line 2)). X-ray irradiation regime is $30 \mathrm{kV}$ and $20 \mathrm{~mA}$.

Crystals growing on the hairs in the cylindrical test-tube with $2 \mathrm{~cm}$ diameter and $4 \mathrm{~cm}$ depth was coursed by evaporation. The not irradiated crystals growing depends on relative supersaturation of solution, size of crystal and solid-liquid interfacial energy in complicated way.

The sizes of not irradiated crystals in Fig. 5 can be approximately presented like some lines depending on crystallization time interval. The growth of crystal in this case depends on diffusion rate and flow of $\mathrm{Na}^{+}$and $\mathrm{Cl}^{-}$ ions into the surface of crystal and their incorporation in the lattice. If relative supersaturation and evaporation stay constant, the crystallization rate is constant also. In this case, flow of ions into the crystal and its size must be approximately proportional to the time of crystallization. We have the essentially different situation for crystallization enhanced by irradiation of X-rays when we have ions diffusion not only in interface region but also in the bulk of growing crystal.

The size of fast growing crystals presented in Fig. 6 in this case is proportional to square root of crystallization time $[7,4]$ and weakly depends on other factors. The number of introduced ions $[7,4]$ in the bulk of growing crystal

$$
N(t)=0.5492 N_{\mathrm{s}} x_{0}, \quad x_{0}=1.616 \sqrt{D t}
$$

is proportional to ions concentration $N_{\mathrm{s}}$ on the surface of crystal and to the square root of the crystallization time and the ions diffusion coefficient $D$. Here, $x_{0}$ is the maximum penetration depth of ions into the crystal, which is proportional to the square root $\sqrt{t}$ of the crystallization time $t$. The diffusion coefficients and crystallization rates can be increased many times [5] by generation of metastable vacancies in growing crystals using X-ray radiation.

The theoretical expression of crystallization rate [13] can be obtained using the spherical nucleus approximation of the Gibbs free energy

$$
\Delta G=\frac{4}{3} \pi R^{2} \rho_{\mathrm{s}} \Delta \mu+4 \pi R^{2} \gamma,
$$

where $\Delta \mu$ is the difference of chemical potential between the liquid and the solid $(\Delta \mu<0)$ and $\gamma$ is the liquid-solid free energy density. $R$ is the nucleus radius. These constants can be obtained from the critical size of nuclei (seeds), corresponding to the beginning of crystallization of the liquid $(\Delta G<0)$, and probabilities to form crystals of different sizes.

The results presented in Fig. 6 are particularly interesting. Crystallization rates are governed by ions diffusion from the solution into the bulk of excited crystals [4]. In this case, an increase of the crystal size in some direction due to crystallization must be proportional to the amount of ions introduced across the orthogonal crystallization surface. This amount is proportional [4] to $\sqrt{D t}$, where the $t$ is the crystallization time and $D$ is the diffusion coefficient. That is why the crystals sizes dependence on $\sqrt{t}$ has been plotted in Fig. 6. Linearity of that dependence confirms that crystal growth is caused by vacancy diffusion into the crystal. During crystallization of $\mathrm{NaCl}$, the diffusion coefficients are significantly increased by irradiated chlorine vacancies. The metastable radicals $\mathrm{H}_{3} \mathrm{O}^{+}$are also important for nucleation like active molecular aggregates whose interaction with negative ions $\mathrm{Cl}^{-}$ can increase ions diffusion flows and crystallization rate. From the data presented in Fig. 4, Fig. 6 for the case of irradiated crystallization and Fig. 3, Fig. 5 for the case of non-irradiated crystallization, we conclude that irradiation can cause an increase of crystallization rates.

\section{Conclusions}

X-ray irradiation causes an increase of the small crystals growth rate of $\mathrm{NaCl}$ crystals in a drop of solution from 2 till 10 times (Fig. 1, Fig. 2). Comparing crystallization in not irradiated (Fig. 5) and in irradiated (Fig. 6) solutions we obtained increasing linear rates of crystallization for lesser crystals $(1.297 \mathrm{~mm}$ and $0.914 \mathrm{~mm}$ consequently, curve 1 and line 1) 200 times and for bigger ( $3.477 \mathrm{~mm}$ and 4.183 consequently, curve 2 and line 2) about 700 times. For non-irradiated case (Fig. 5) crystallizations rates for lesser and bigger crystals practically coincide and approximately equal $0.00015 \mathrm{~mm} / \mathrm{h}$. For crystallization with irradiation (Fig. 6) we obtained linear crystallization rate $0.034 \mathrm{~mm} / \mathrm{h}$ for lesser crystal and for bigger $0.097 \mathrm{~mm} / \mathrm{h}$. From obtained results, we can confirm that dividing crystallization rates and increasing of sizes by crystallization we obtain the same 2.9 relation. The increase of crystallization rate in bigger crystal is based on irradiation of more vacancies taking part in crystal growing. The linear dependence of crystals size (Fig. 6) on $\sqrt{t}[4,14]$ indicates that in the rate of crystallization processes most important place take the ions diffusion by irradiated vacancies in the crystal bulk.

Due to ions diffusion by vacancies created by X-ray radiation, the ions deposited from the solution onto the crystal surface can diffuse into the bulk much faster. The rate of growth of the new crystals in drop a non-irradiated 
solution (Fig. 2) decreases with increasing size of the crystals, whereas in an irradiated solution (Fig. 1) this decrease has been observed until the crystal size reaches $\approx 0.23 \mathrm{~mm}$. Further on, their growth rate increases by irradiated vacancies density increasing in the growing crystal.

\section{References}

[1] J. Ulrich, C. Strege, J. Cryst. Growth 237-239, 2130 (2002).

[2] S.-M. Park, J.M. Kim, H.Ch. Lee, Ch.-K. Kim, Jpn. J. Appl. Phys. 35, 1554 (1996).

[3] A.J. Janavičius, Lith. J. Phys. 37, 508 (1997).

[4] A.J. Janavičius, Acta Phys. Pol. A 93, 505 (1998).

[5] A.J. Janavičius, S. Balakauskas, V. Kazlauskiene, A. Mekys, R. Purlys, J. Storasta, Acta Phys. Pol. A 114, 779 (2008).

[6] J. Sharma, R. Smoluchowski, Phys. Rev. 137, A259 (1965).
[7] V.I. Dubinko, D.I. Vainshtein, H.W. den Hartog, Nucl. Instrum. Methods Phys. Res. B 228, 304 (2005).

[8] F.S. Dainton, Br. J. Radiol. 24, 428 (1951).

[9] R. Karazija, The Theory of X-ray and Electronic Spectra of Free Atoms. An Introduction, Mokslas, Vilnius 1987, p. 274 (in Russian).

[10] A.J. Janavičius, R. Purlys, A. Bulkšas, S. Balakauskas, A. Mekys, V. Šablinskas, Acta Phys. Pol. A 116, 1076 (2009).

[11] J. Murray Gibson, Mater. Sci. 326, 942 (2009).

[12] W. Chen, C. Tegenkamp, H. Pfnür, Phys. Rev. B 82, 104106 (2010).

[13] S. Auer, D. Frenkel, J. Chem. Phys. 120, 3015 (2004).

[14] A.J. Janavičius, Acta Phys. Pol. A 93, 731 (1998). 